\title{
Cost Comparison of Single-Use Versus Reusable Bronchoscopes Used for Percutaneous Dilatational Tracheostomy
}

\author{
Anne Sohrt ${ }^{1} \cdot$ Lars Ehlers $^{2} \cdot$ Flemming Witt Udsen $^{3} \cdot$ Anders MærkedahI $^{1}$ (1) $\cdot$ Brendan A. McGrath $^{4}$
}

Published online: 30 August 2018

(c) The Author(s) 2018

\begin{abstract}
Background Both single-use and reusable bronchoscopes are suitable for percutaneous dilatational tracheostomy (PDT) to visualise the trachea during the insertion process. To determine the least costly option, the price of single-use bronchoscopes must be weighed against the estimated average cost of a bronchoscopy with reusable equipment. In the latter case, the acquisition cost must be spread over the equipment's useful life and other relevant costs, such as reprocessing and repair, must be included.

Objective This study aimed to calculate the cost of using single-use or reusable bronchoscopes per PDT procedure.

Methods A systematic literature search was conducted to identify studies comparing the costs of reusable and single-use bronchoscopes for PDT. Inclusion criteria were articles assessing the cost of single-use or reusable bronchoscopes, and where costs were divided into acquisition, reprocessing, and repair costs. A questionnaire regarding repair rates and costs for reusable bronchoscopes was sent to 366 hospitals in the US, UK, and Germany to supplement the identified literature.

Results Eleven studies met the inclusion criteria. Ninety-nine completed responses were received, of which 31 hospitals used reusable equipment for PDT. Literature research revealed an average acquisition cost of \$US135 (SD 152) and reprocessing cost of \$US123 (SD 128). Additionally, a combination of data from the literature and the questionnaires gave a repair cost per use of \$US148 (SD 242), resulting in a total average cost of \$US406 for reusable bronchoscopes and \$US249 (SD 36) for single-use bronchoscopes per PDT procedure. Thus, the incremental cost per use of a reusable bronchoscope compared with a single-use bronchoscope was \$US157.

Conclusions We conclude that significant savings can be made by using single-use bronchoscopes to guide PDT in preference to reusable bronchoscopes. Results depend on hospital setting, the reprocessing procedures, annual bronchoscope procedures, individual repair cost, and repair rates.
\end{abstract}

\section{Key Points for Decision Makers}

The study indicates that significant savings can be made

Electronic supplementary material The online version of this article (https://doi.org/10.1007/s41669-018-0091-2) contains supplementary material, which is available to authorized users.

Anne Sohrt

andm@ambu.com

1 Ambu A/S, Baltorpbakken 13, 2750 Ballerup, Denmark

2 Department of Business and Management, Fibigerstraede 11, 9220 Aalborg, Denmark

3 Department of Health Science and Technology, Frederik Bajers Vej 7, 9220 Aalborg, Denmark

4 Wythenshawe Hospital, Manchester University Hospital NHS Foundation Trust, Southmoor Road, Manchester M23 9LT, UK by using single-use bronchoscopes to guide percutaneous dilatational tracheostomy (PDT) in preference to reusable bronchoscopes.

Estimated cost savings associated with the use of singleuse bronchoscopes was \$US157 per PDT procedure.

Our findings are associated with a certain degree of uncertainty due to the increased variances in the findings from the literature. 


\section{Introduction}

Tracheostomy involves the creation of an artificial stoma between the trachea and the anterior neck. Whilst classically performed as an open surgical procedure by head and neck surgeons to relieve actual or threatened airway obstruction, around two-thirds of tracheostomies are currently performed by intensive care unit (ICU) clinicians on critically ill patients. The majority of these procedures are performed to facilitate weaning from prolonged mechanical ventilation [2]. Percutaneous dilatational tracheostomy (PDT) is an alternative tracheostomy insertion technique that can be performed at the bedside, and is an attractive option when considering the practical and logistical challenges of moving a critically ill patient to an operating theatre [3, 4]. The PDT technique was described by Ciaglia in 1985 , and has subsequently become widely used as a practical, safe and cost-effective alternative to open surgical tracheostomy [3-6]. The procedure involves the insertion of a needle through the skin of the anterior neck into the trachea, followed by the insertion of a guidewire. Using a 'Seldinger technique', serial dilators are then guided into the trachea via the guidewire to create the stoma before the tracheostomy tube is finally inserted [3-7].

PDT is usually performed with bronchoscopic guidance to visualise the trachea during the insertion process $[2$, 7-9]. Both single-use and reusable bronchoscopes are suitable for this procedure [1]. Single-use bronchoscopes are disposed of after the procedure, whereas reusable devices require cleaning, decontamination, sterilisation and sterile storage, with further re-sterilisation determined by the duration of storage. To estimate the cost per use for reusable bronchoscopes, the acquisition cost must be spread over the equipment's useful lifetime (which is not known in advance) and costs associated with reprocessing, storage and repair must be included. Many reusable devices also require a separate light source, an image capture, and display system, although these can be shared between multiple devices. Bronchoscopes may be damaged during PDT procedures, in which a needle is inserted in close proximity to the device [1]. One hospital reported six perforations of reusable bronchoscopes occurring during 42 PDT procedures from January 2007 to November 2010, leading to a total cost of repair of around \$US10,700 [10]. Perbet et al. estimated the repair costs of bronchoscopes damaged by PDT to be \$US475 per use (based on 76 PDT procedures from August 2009 to July 2014) [11]. Bronchoscopes can also be damaged during other procedures or by handling, processing or storage. The repair cost following bronchoalveolar lavage (BAL) procedures has been reported to be \$US57 (based on 381 BAL procedures) [11].
The cost per use for single-use bronchoscopes is the actual acquisition cost. Typically, prices for medical devices are likely to change over time due to the market entry of new products and innovation and may vary between countries and healthcare systems according to the way devices are procured (e.g. scale advantages) [12].

The choice of single-use or reusable bronchoscopes for PDT is multifactorial, but cost is important to any organisation. Therefore, the aim of this study was to calculate the cost of use of single-use or reusable bronchoscopes per PDT procedure from a hospital perspective, based on the available literature, supplemented by questionnaire data from end users where necessary.

\section{Methods}

\subsection{Literature Search}

A systematic literature search, using a PICO approach, was conducted to identify articles assessing the cost of singleuse and reusable bronchoscopes. Keyword searches were performed using the Cochrane database, PubMed, Embase, and Google Scholar, using search terms [(bronchoscope OR bronchoscopes) AND cost AND (reusable OR single-use)]. The search was limited to articles published from 1 January 2012 to 21 September 2017, since the first single-use bronchoscope was launched in 2012, and to human subjects. The systematic search was supplemented by an internet search using the Google search engine to identify posters and reports assessing the cost of bronchoscopes. A manual review of abstracts for the identified papers was carried out by AS and screened against the following inclusion criteria: articles assessing the cost of single-use or reusable bronchoscopes, and where costs were divided into acquisition, reprocessing and repair costs.

We coped with selection bias by using a structured method for the literature search (PICO), included grey literature, no language restriction and additional review of excluded and included literature by co-author AM.

For all included papers, a retrospective unit-cost approach was used, for example hospital register data, bronchoscope tracking systems and procedure flow data. Tvede et al. was the only study that included micro-costing via prospective time measurement for personal time spent on individual steps in the cleaning procedures [13].

Procedures investigated were tube intubation for airway management, BAL, bronchoalveolar wash, difficult airway management, secretion management, and PDT procedures.

Acquisition cost and reprocessing cost per bronchoscope use were extracted for all included papers. The total cost associated with the reusable bronchoscopes, including all essential equipment, such as rack systems, light sources and 
image capture, was divided by the number of procedures, number of bronchoscopes available and the expected lifetime of the bronchoscope estimated in the studies. The cost of use of single-use bronchoscopes included the acquisition cost of the bronchoscope and the associated monitor, which was calculated according to the specific need for monitors per hospital. All costs were estimated in 2016 prices and the calculated costs were presented as means (standard deviations) in United States Dollars (\$US). Costs that were not obtained in 2016 prices were projected to 2016 values using the consumer price index from Trading Economics [15]. Only costs that could be directly attributed to the use of bronchoscopes and costs that differed between single-use and reusable bronchoscopes were included. It was assumed that staffing and consumable costs related to conducting the procedure did not differ between devices.

\subsection{Questionnaire}

In most studies included in the literature search, the cost calculations were based on BAL or unspecified procedures. To gather PDT-specific data, a questionnaire regarding repair rates and the costs of reusable bronchoscopes used for PDT was conducted to supplement the estimation from the literature. Due to scarce resources, western countries were selected. US, UK and Germany were selected as they represent the biggest markets for single-use bronchoscopes and collectively represent population majority [14]. The questionnaire was sent to 366 hospitals in the US, UK and Germany that were known to perform PDT. The repair cost per bronchoscope use was calculated based on the total number of procedures, the total number of times the bronchoscopes had been damaged and the mean repair cost. A copy of the questionnaire is available in the Electronic Supplementary Material.

The mean repair cost per bronchoscope use for this study was calculated from questionnaire results and from Perbet et al. [11] weighted by the number of procedures reported.

\subsection{Sensitivity Analyses}

Due to variance in cost estimations among the studies identified from the literature, and to make the data generalisable to other hospitals, two-way sensitivity analyses were conducted to test the relationship between the cost of reusable and single-use bronchoscopes in two different scenarios. For the first analysis, acquisition and reprocessing costs for reusable devices were pooled and set to \$US150, demonstrating a conservative approach. In the second analysis, acquisition and reprocessing costs were set to \$US207 as this is the accumulated mean when outliers are removed. For both analyses, the cost per repair was varied from \$US1000 to \$US6000 and the repair ratio was varied from 5 to $8 \%$.
Furthermore, as the price for single-use bronchoscopes can vary between countries and depends on the number of bronchoscopes purchased, a one-way analysis was conducted. The price was adjusted from the lowest to the highest price from the studies identified in the literature (\$US208-322).

\section{Results}

A total of 422 articles were retrieved and abstracts were screened (Fig. 1). Seven articles were identified as relevant: six assessing the cost of both single-use and reusable bronchoscopes and one assessing the cost of reusable bronchoscopes only. Furthermore, four relevant posters were identified by an additional Google search.

The mean cost per use of a reusable bronchoscope was estimated based on these 11 studies, describing 4476 procedures. The studies were from the US, UK, France and Denmark and were published between 2011 and 2017 (see Table 1). The mean acquisition cost for a reusable bronchoscope was calculated to be \$US135 (SD 152) per use, with a mean reprocessing cost of $\$$ US123 (SD 128) per use [11, 13, 16-24]. Based on 10 studies, the cost per use of a single-use bronchoscope was estimated to be \$US249 (SD 36) [11, 13, 16-19, 21-24].

Ninety-nine completed responses were received from the 366 questionnaires (27\% response rate). Of these, 31 hospitals used reusable equipment; 20 (64.5\%) from the US, 8 (25.8\%) from the UK and 3 (9.7\%) from Germany, with a total annual number of 1698 PDT procedures performed. A repair ratio of 1:27 (corresponding to 3.7\%) was associated with a mean repair cost of \$US3530 (SD 2669), giving a mean repair cost per PDT use of \$US133. The weighted mean repair cost following PDT from Perbet et al. [11] and the questionnaires was calculated to be \$US148.

The total cost per use of a reusable bronchoscope for PDT was calculated to be $\$$ US406 by combining acquisition, reprocessing and weighted mean repair costs. With the

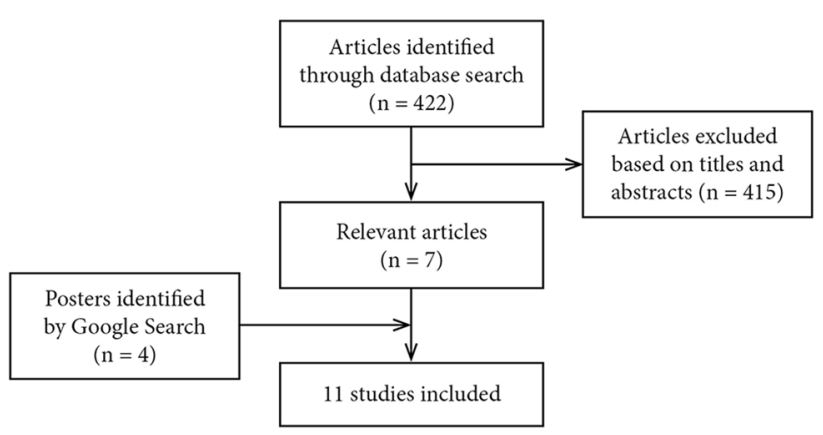

Fig. 1 Study selection process 


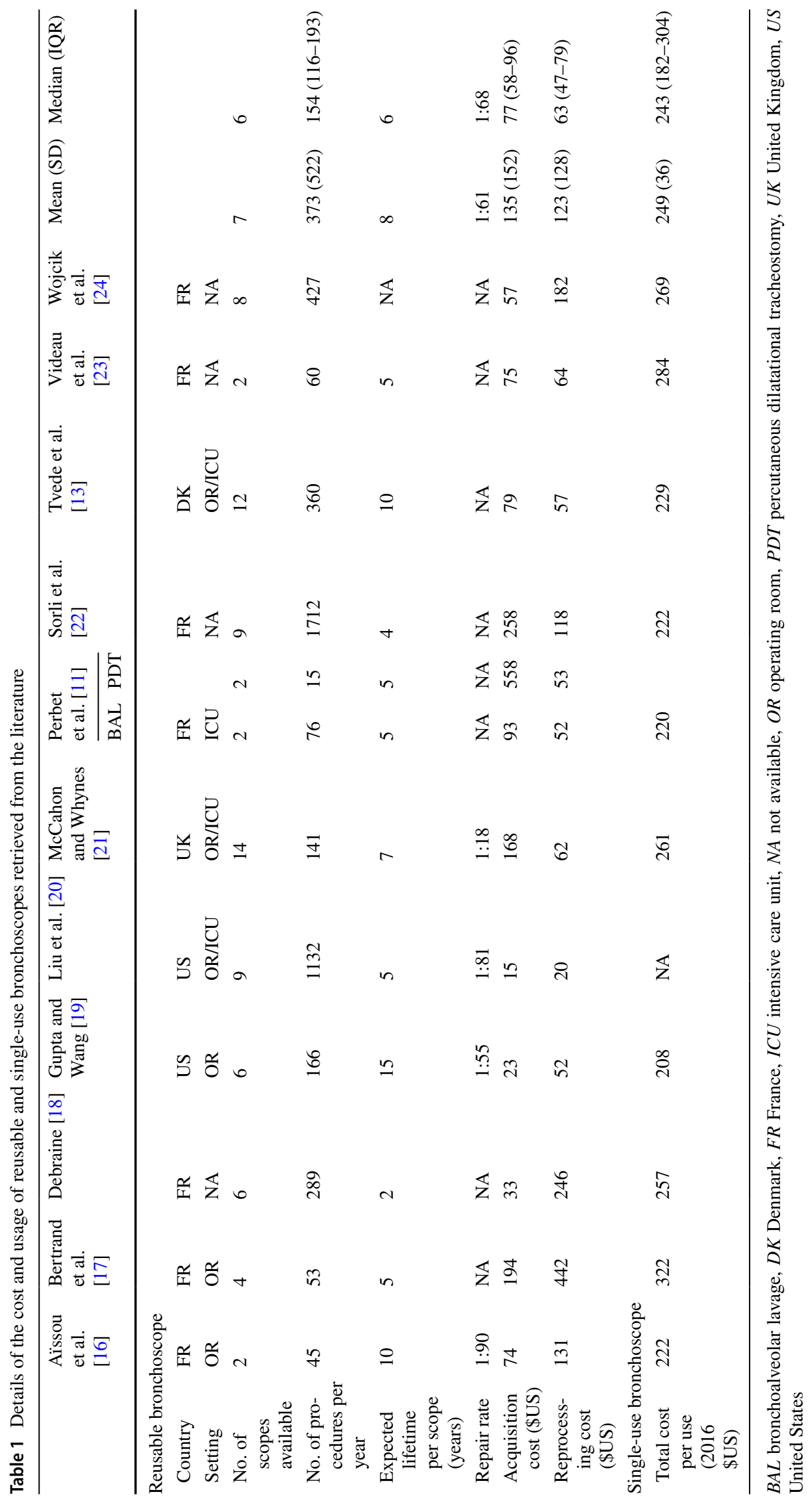


cost of a single-use bronchoscope estimated to be \$US249 per use, the incremental cost per use of a reusable bronchoscope compared with a single-use bronchoscope was \$US157. This equates to a $39 \%$ saving per PDT procedure for single-use bronchoscopes when compared with reusable bronchoscopes.

The two-way sensitivity analyses indicated potential cost savings in different scenarios for the reusable bronchoscopes for PDT. Using a conservative approach accumulating acquisition and reprocessing costs to \$US150, the use of reusable bronchoscopes results in cost savings when repair costs and repair rates are below \$US3530 and 3.7\%, respectively.

The higher cost per repair and repair rate for reusable bronchoscopes made the single-use technology more advantageous (Fig. 2). The one-way sensitivity analysis using the range of identified purchase costs for single-use bronchoscopes demonstrated that single-use devices remained cheaper than reusable devices.

\section{Discussion}

This study examined the average cost of bronchoscope use associated with PDT procedures using a combination of the available literature and questionnaires, finding that singleuse devices were cheaper than reusable devices. These findings have implications for the procurement of bronchoscopes by hospitals and units that perform PDT. The two-way sensitivity analyses confirmed that the single-use device led to cost savings even in scenarios where several cost parameters for the reusable device were lowered.

Our study was the first to investigate in detail repair rates and associated costs for bronchoscopes specifically for PDT. The questionnaire data confirmed our prior assumptions that reusable bronchoscope repair rates were higher for PDT procedures than other bronchoscopy procedures. Four of the

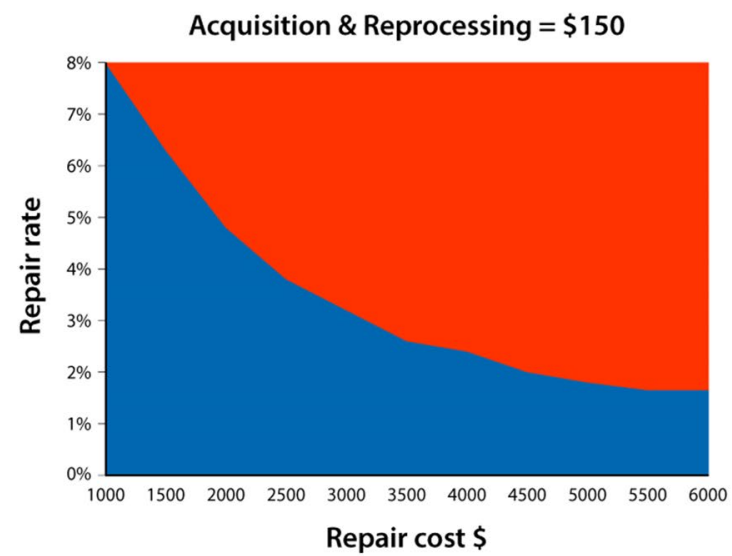

studies we identified reported repair rates for bronchoscopy procedures in general, with a pooled mean repair rate of 1 in 61 uses (Table 1) [16, 19-21]. This was significantly lower than the 1 in 27 repair rate for PDT procedures from the questionnaire. Furthermore, the repair cost per use for PDT identified in this study was higher than the repair cost for bronchoscopy procedures in general reported in the literature, perhaps reflecting more significant damage to reusable devices associated with the PDT procedure.

The identified studies revealed large differences in average costs per PDT for reusable bronchoscopes. One explanation for this could be a scale advantage (the negative associations identified between the number of annual procedures and reusable procedural costs) (Table 1). Secondly, reprocessing of bronchoscopes is complex and time consuming. New US standards collectively recommend $>100$ steps for reprocessing each endoscope [25-29]. Only one study conducted accurate time measurements for personal time spent on reprocessing [20]. Further, data from included studies are collected across countries and in different hospital settings, causing variance in reprocessing requirements, equipment acquisition costs, repair ratio and unit-costing method. For instance, in Australia and France reprocessing guidelines recommend re-cleaning of endoscopes if not used after $12 \mathrm{~h}$, leading to a significant increase in costs [30].

Thirdly, in several studies the economic calculations were inadequately described and therefore methodological differences cannot be excluded.

The previous studies only presented mean costs without dispersions and uncertainty measures. Thus, it was difficult to conduct meta-analyses on their results. In this study, we used the mean cost reported in the previous studies as this reflected the large variation in the annual number of procedures. Due to the variety of methodologies available to examine these figures, it could be argued that median values better reflect the 'typical' cost per use. This would eliminate

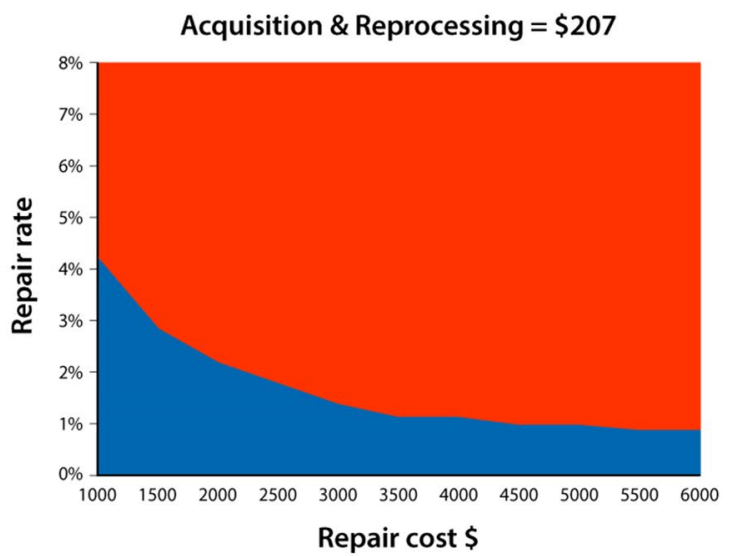

Fig. 2 Two-way sensitivity analyses: the red area indicates savings from using single-use bronchoscopes and the blue area indicates savings from using reusable bronchoscopes. Costs are in 2016 US dollars 
the influence of the outliers and the cost of reusable bronchoscopes would be decreased. The median acquisition cost from the literature was \$US77 (15-558) and the median reprocessing cost was \$US63 (20-442). Together with the median repair cost estimated in this study (\$US129), the total cost per reusable bronchoscope calculated using median values was \$US269, while the median cost per use for single-use bronchoscopes was \$US243 [11, 13, 16-19, 21-24]. Thus, with median costs estimates, the incremental cost per use of a reusable bronchoscope compared with a single-use bronchoscope is \$US26. This is significantly lower in comparison with when mean values are used. A more ideal approach to estimate the true cost of use of reusable bronchoscopes would be to make a micro-costing analysis based on a suitable number of hospitals.

The majority of completed questionnaire responses are from the US, hence this can have an impact on average repair cost, since the US is known to have higher healthcare expenses [31]. However, when calculating the individual mean from the US responses, it is in line with the German responses: \$US4275 and \$US4285, respectively. UK mean repair cost, on the other hand, appears significantly lower with \$US1757 per repair.

For single-use bronchoscopes, the scale advantage might also have an impact on the price because it is often possible to receive a discount when a large number of devices are purchased. Currently, there are only a few producers in the market manufacturing single-use devices. It is possible that more producers would increase competition in the market and that the price would be reduced, consequently making the single-use devices even more advantageous.

There may also be additional advantages in using disposable equipment, which may also have direct or indirect cost implications, such as the elimination of bronchoscope crossinfections and immediate availability in an emergency situation [9, 32, 33]. A recent article by Terjesen et al. estimated additional costs associated with treatment of bronchoscope cross-infections to be \$US203 per procedure [33].

The costs of the differences in time required to complete the procedure and number of personnel assisting per procedure have not been included in the study. The delivery and preparation of ancillary equipment to facilitate a reusable bronchoscopy have been shown to delay the start time of the procedure when compared with using single-use bronchoscopes [32]. In contrast, the sensitivity analyses demonstrated scenarios where reusable bronchoscopes had the cost advantage; for example, when repair cost and repair ratio were below \$US3530 and 3.7\%, respectively, in combination with acquisition and reprocessing costs equal to \$US150 per use or below.

The use of single-use bronchoscopes is associated with directly increased waste disposal and handling costs for the procedure. However, the waste cost for single-use bronchoscopes is estimated to be between \$US0.05 and \$US0.06 [11, 18]. Furthermore, the reprocessing of reusable bronchoscopes is associated with waste disposal such as single-use cleaning and protection equipment, disinfectants and chemical disposal. The cost of wage management has no impact on the conclusion.

\section{Conclusion}

Our study suggests that significant savings can be made by using single-use bronchoscopes to guide PDT in preference to reusable devices, and these findings may also be relevant for bronchoscope-guided procedures other than PDT. Further, we believe the findings are generalisable in Europe and the US. Finally, understanding institutional bronchoscope use and the associated costs in general and for PDT will enable an informed assessment of which approach represents better value for a particular unit or service.

Data Availability Statement Acopy of the questionnaire, questionnaire results, and cost data is available in the Electronic Supplementary Material.

Author contributions AS: data collection and writing of the manuscript. LE, FWU, AM, and BAM: methodology advice and manuscript drafting and revision. All authors approved the manuscript.

\section{Compliance with Ethical Standards}

Funding This study was funded by Ambu A/S.

Conflict of interest AS and AM are employed by Ambu. BAM has received expenses from Ambu for attending company educational and product evaluation events, for which he has declined personal payment. BAM has also received bronchoscopes and loan equipment free of charge from Ambu and Olympus KeyMed to support research studies. For the remaining authors, none were declared.

Open Access This article is distributed under the terms of the Creative Commons Attribution-NonCommercial 4.0 International License (http://creativecommons.org/licenses/by-nc/4.0/), which permits any noncommercial use, distribution, and reproduction in any medium, provided you give appropriate credit to the original author(s) and the source, provide a link to the Creative Commons license, and indicate if changes were made.

\section{References}

1. NICE. Ambu aScope 2 for use in unexpected difficult airways. 2013. https://www.nice.org.uk/guidance/mtg14. Accessed 20 Aug 2018 .

2. Wilkinson KA, Martin IC, Freeth H, Kelly K, Mason M. On the right trach? 2014. National Confidential Enquiry into Patient 
Outcome and Death (NCEPOD), UK. https://www.ncepod.org. uk/2014tc.html or to full report https://www.ncepod.org.uk/2014r eport1/downloads/OnTheRightTrach_FullReport.pdf.

3. Ciaglia P, Firsching R, Syniec C. Elective percutaneous dilatational tracheostomy: A new simple bedside procedure; preliminary report. Chest. 1985;87:715-9.

4. Barba CA, Angood PB, Kauder DR, Latenser B, Martin K, Mcgonigal MD, et al. Bronchoscopic guidance makes percutaneous tracheostomy a safe, cost-effective, and easy-to-teach procedure. Surgery. 1995;118:879-83.

5. Cabrini L, Monti G, Landoni G, Biondi-Zoccai G, Boroli F, Mamo D, et al. Percutaneous tracheostomy, a systematic review. Acta Anaesthesiol Scand. 2012;56:270-81.

6. Johnson-Obaseki S, Veljkovic A, Javidnia H. Complication rates of open surgical versus percutaneous tracheostomy in critically ill patients. Laryngoscope. 2016;126:1-9.

7. Madsen KR, Guldager H, Rewers M, Weber SO, Købke-Jacobsen K, White J. Danish Guidelines 2015 for percutaneous dilatational tracheostomy in the intensive care unit. Dan Med J. $2015 ; 62(3): 1-8$

8. Gadkaree SK, Schwartz D, Gerold K, Kim Y. Use of bronchoscopy in percutaneous dilational tracheostomy. JAMA Otolaryngol Head Neck Surg. 2016;142:143-9.

9. Cook T, Woodall N, Frerk C. Major complications of airway management in the United Kingdom. 4th National Audit Project of The Royal College of Anaesthetists and The Difficult Airway Society. Report and findings; 2011. https://www.rcoa.ac.uk/nap4 or full report https://www.rcoa.ac.uk/system/files/CSQ-NAP4Full.pdf.

10. Jackson LSM, Davis JW, Kaups KL, et al. Percutaneous Tracheostomy : To Bronch or Not to Bronch - That Is the Question. J Trauma Acute Care Surg. 2011;71:13-6.

11. Perbet $\mathrm{S}$, Blanquet $\mathrm{M}$, Mourgues $\mathrm{C}$, et al. Cost analysis of singleuse (Ambu ${ }^{\circledR}$ aScope ${ }^{\mathrm{TM}}$ ) and reusable bronchoscopes in the ICU. Ann Intensive Care. 2017;7:3.

12. Drummond MF, Griffin A, Tarricone R. Economic evaluation for devices and drugs. Same or different? Value Health. 2009;12:402-4.

13. Tvede MF, Kristensen MS, Nyhus-Andreasen M. A cost analysis of reusable and disposable flexible optical scopes for intubation. Acta Anaesthesiol Scand. 2012;56:577-84.

14. Ambu A/s annual report 2017. https://www.ambu.com/about/ corporate-info/investors/reports/reports-in-english. Accessed 27 Aug 2018.

15. Trading Economics. Consumer price index 2017. http://www. tradingeconomics.com/united-states/consumer-price-index-cpi. Accessed 1 Nov 2017.

16. Aïssou M, Coroir M, Debes $\mathrm{C}$, et al. Analyse de coût comparant les fibroscopes à usage unique $\left(\mathrm{Ambu}^{\circledR} \mathrm{aScope} \mathrm{T}^{\mathrm{TM}}\right)$ et les fibroscopes réutilisables pour l'intubation difficile. Ann Fr Anesth Reanim. 2013;32:291-5.

17. Bertrand A, Lefrançois A, Saurel N. Etude de cout en faveur du fibroscope á usage unique. Europharmat 2014.
18. Debraine. Fibroscope réutilisable versus usage unique : analyse de coûts en réanimation. XXVII Congrès Natl la Société Française d'Hygiène Hosp 2016.

19. Gupta D, Wang H. Cost-effectiveness analysis of flexible optical scopes for tracheal intubation: a descriptive comparative study of reusable and single-use scopes. J Clin Anesth. 2011;23:632-5.

20. Liu SS, Brodsky JB, Macario A. Cost identification analysis of anesthesia fiberscope use for tracheal intubation. J Anesth Clin Res. 2012;3:3-6.

21. McCahon RA, Whynes DK. Cost comparison of re-usable and single-use fibrescopes in a large English teaching hospital. Anaesthesia. 2014;70:699-706.

22. Sorli SC, C DFF, Thiveaud D, Pecani D, Pôle CHUT, Logipharma P. Etude de coût des fibroscopes réutilisables vs jetables en réanimation. Europharmat. 2015.

23. Videau M, Rghioui K, Mottet B, Sainfort A, Lefort I. Analyse comparative de coût entre les fibroscopes bronchiques à usage unique et réutilisables : le fibroscope à usage unique, est-ce que ca vaut le coût? Ann Fr Anesth Reanim. 2017;75:473-9.

24. Wojcik A, Tywoniuk M, Vella I, Luyckx M. Medico-economic benefit from replacing reusable bronchoscopes with single-use versions: a microcosting evaluation; 2015. p. 314. http://www. sf2s-sterilisation.fr/wpcontent/uploads/2016/08/ID122_WFHSS _\%202015.pdf.

25. Association for the Advancement of Medical Instrumentation. ANSI/AAMI Standard 91: Flexible and semi-rigid endoscope processing in health care facilities. 2015; pp 1-70.

26. AORN. Guideline for processing flexible endoscopes. 2016; pp 675-758. https://aornguidelines.org/guidelines/. Accessed 20 June 2018.

27. SGNA. Standard of infection prevention in the gastroenterology setting. 2015. https://www.sgna.org/Practice/Standards-PracticeGuidelines. Accessed 20 Aug 2018.

28. CDC. Guideline for disinfection and sterilization in healthcare facilities. 2008. https://www.cdc.gov/infectioncontrol/guidelines /disinfection/index.html. Accessed 20 Aug 2018.

29. Ofstead CL et al. A glimpse at the true cost of reprocessing endoscopes: results of a pilot project; 2017. http://iahcsmm.org. Accessed 13 Apr 2018.

30. Choi H, Cho YS. Endoscope reprocessing: update on controversial issues. Clin Endosc. 2015;48(5):356-60.

31. Kaiser Family Foundation analysis of data from OECD. OECD Health Data: health expenditure and financing: Health expenditure indicators. OECD Health Stat (database). 2017. https://doi. org/10.1787/health-data-en (accessed on March 19).

32. Marshall DC, Dagaonkar RS, Yeow C, et al. Experience with the use of single-use disposable bronchoscope in the ICU in a tertiary referral center of Singapore. J Bronchol Interv Pulmonol. 2017:24:136-43.

33. Terjesen CL, Kovaleva J, Ehlers L. Early assessment of the likely cost effectiveness of single-use flexible video bronchoscopes. PharmacoEconomics. 2017;1:133-41. 